\title{
MODELOS MACROECONOMÉTRICOS PARA FORMULAR POLITICAS DE DESARROLLO Y PLANEACIÓN
}

\author{
JEFFREY B. NUGENT
}

\section{INTRODUCCIÓN}

EN LOS ÚlTIMOS quince años se han presenciado dramáticos avances en el desarrollo de los modelos de planificación a largo plazo y de criterios prácticos para ubicar recursos en el mercado competitivo característico de la mayoría de los países menos desàrrollados (PMD). En contraste, la construcción y uso de modelos macroeconómicos de corto plazo para políticas de planeación, es relativamente primitivo en el contexto de los PMD, con formas elementales calcadas de modelos econométricos más antiguos desarrollados en los países desarrollados (PD).

En la sección siguiente, se pondrá énfasis en los desarrollos recientes que están comenzando a lograr la adecuación entre los modelos utilizados tanto con las características de los PMD como con las necesidades de los planificadores. Al mismo tiempo, se especula acerca de la natuleza de las innovaciones que será necesario hacer antes que los PMD puedan tener modelos macroeconométricos satisfactorios.

En la segunda sección, se presenta un método de aplicación de modelos macroeconométricos a políticas de planeación con el uso de técnicas de programación lineal. El uso de la programación lineal en políticas de planeación, es presentado en el contexto de la coordinación de una política regional (multinacional) entre países de América Central. Se específica un modelo de pequeña escala y luego se estima separadamente para cada uno de los cinco países de América Central. Los modelos son acoplados conjuntamente para formar un gran modelo econométrico para la región. La programación lineal es entonces aplicada al modelo para resolver el conjunto óptimo de políticas macroeconómicas bajo las dos estructuras institucionales alternativas - primero sin considerar coordinación entre países y luego considerando una coordinación completa. Una comparación de estas soluciones proporciona una medida de los beneficios potenciales de una mayor coordinación de políticas. El trabajo concluye con algunas sugestiones para investigación institucionalizada acerca del diseño de modelos macroeconométricos de los PMD. 


\section{TENDENCIAS PASAdAS Y FUTURAS DE LOS MODEloS MACROECONOMÉTRICOS}

¿Qué clase de modelos deben emplearse en los PMD? Esta pregunta, planteada por Lawrence Klein hace casi diez años (Klein, 1965), se encuentra abierta a pesar de que en el decenio presente se han hecho serios intentos, por parte del mismo Klein, de algunos de sus discípulos $c f$. y colegas, y otros, por adelantar algunas respuestas. ${ }^{1}$

Como observó Klein, los primeros modelos para los PMD (cf. Narashimham, 1956; Suits, 1964) siguieron un enfoque muy cercano al del modelo simple keynesiano que había prevalecido en los Estados Unidos y otros PD en los años 40 y 50 . Paulatinamente, las clases de modelos propuestos para los PMD, han comenzado a cambiar. Algunos de los modelos más recientes para PMD han seguido en forma paralela la tendencia reciente de los modelos de PD hacia formulaciones más neoclásicas, con especial énfasis en el papel de los precios relativos y de la maximización del beneficio (Zarembka, 1967, 1972; Marwah, 1963, 1970) y en hacer uso más amplio de las variables de espectativas basadas en formulaciones de retardos distribuidos (Evans, 1970). Otros cambios en los modelos de PMD reflejan cambios en la importancia de sectores diferentes. Mientras que los primeros modelos estuvieron inspirados en los esquemas de economía cerrada de Harrod-Domar y Keynes, el gran papel que el comercio exterior juega en la mayoría de los PMD, se refleja cada vez más en las formulaciones recientes.

Sin embargo, algunas de las innovaciones recientes en los modelos de PMD, representan rupturas más serias respecto a la tradición de los modelos de PD. Mientras que los primeros eran (en la tradición keynesiana) de determinación de demanda, algunos de los más recientes de los PMD, ponen énfasis en consideraciones de oferta (Marwah, 1970; Beltrán del Río y Klein, 1973). En otras desagregaciones se ha permitido la especificación de diferentes funciones de producción y de consumo para sectores diferentes (Islam, 1965; Agarwala, 1970; Zarembka 1972; Kelley, Williamson y Cheetham, 1972), con lo cual al incorporar las nociones de Nurkse-Lewis-Fei-Ranis sobre excedente agrícola y, admitidas distintas clases de imperfecciones de mercado, en algunos modelos persisten diversas formas de dualismo. Con objeto de dar mayor atención al detalle, los modelos más recientes de PMD, por lo general son más adecuados que sus predecesores en reflejar las condiciones especiales e institucionales de los países para los que han sido diseñados. ${ }^{2}$

Sin duda son deseables estas tendencias hacía una mayor diferenciación entre el conjunto de modelos de PMD y entre los de los PD así como entre los diferentes modelos individuales para PMD. Mas aún, es pro-

1 Véase, en especial, del Río y Klein, 1973.

- Desde este punto de vista, véanse los modelos más detallados para México, Beltran del Rio y Klein, 1973; para India, Agarwala, 1970; para Argentina, Nugent, 1967; y para Indonesia, Fukuchi, 1973. 
bable que estos cambios continuen y se aceleren y que, en los siguientes años, los modelos macroeconométricos de estos países alcancen un grado explicativo mucho mayor y utilicen como determinantes variables que hasta ahora han sido excluidas, tales como los factores demográficos, factores estructurales, así como los determinantes y los efectos de los cambios en la distribución del ingreso. Aún en cualquier PMD se puede encontrar un grado creciente de individualidad y diferenciación entre modelos macroeconométricos, que reflejan los crecientes usos diferenciados para los cuales se habilitan así como las distintas "visiones" de los diseñadores de tales modelos.

Muchos de estos cambios esperados se refieren al alcance, nivel de agregación y al énfasis, cambios que en su mayor parte están ya en camino de realizarse. El interés de este trabajo es, además sobre otras diversas áreas en las cuales son necesarias algunas mejoras que, sin embargo, no aparecen muy claras en el horizonte. Estas son: 1) la necesidad de desarrollar de manera más completa y explícita la naturaleza del desequilibrio de las estructuras y del proceso del desarrollo; 2) la necesidad de integrar las perspectivas de corto, mediano y largo plazo y; 3) la necesidad de relacionar estos modelos de manera más cercana con los requerimientos de la planificación del desarrollo y con las decisiones de política.

\section{Desequilibrio}

Principiamos con el desequilibrio. Cada día más y más economistas reconocen que vivimos en un mundo menos que perfecto y en el cual a menudo el óptimo, el "primero-mejor", y aun el mecanismo de ajuste del "segundo-mejor" no pueden ser utilizados en los impracticables campos de la política o de la administración. Con los mecanismos de ajuste óptimo descartados u obstaculizados por las restricciones de política, y otros desechados de los terrenos económicos y sociales, las diversas diferencias dualísticas observadas entre un sector y otro, de hecho tienden a permanecer $y$, en algunos casos, se acentúan a medida que transcurre el tiempo y tiene lugar el desarrollo. ${ }^{3}$ Esto puede ser resultado, en parte, de políticas imperfectas, lo que sugiere la necesidad (que se estudia luego) de modelos macroeconométricos para PMD capaces de producir implicaciones más útiles de política. Sin embargo, esto parece deberse parcialmente a la relativa intensidad de diversas fuerzas desequilibrantes que al parecer se producen de manera natural en el proceso del desarrollo económico.

El descuido de los precios, de la tecnología, de los hábitos de ahorro y de las existencias de capital humano y de sectores que conducen al

\footnotetext{
${ }^{3}$ Este tema es desarrollado con detenimiento en Yotopoulos y Nugent (de próxima publicación).
} 
equilibrio, es un hecho importante de la vida en los PMD, lo que sugiere la euidadosa consideración que debe prestarse a la naturaleza y magnitud de los factores que impiden alcanzar el equilibrio y el rango de validez de cualquier restricción particular. Por ejemplo, en relación al mercado de un bien individual, caracterizado por exceso de oferta o de demanda, olvidar el ajuste de los precios a un nivel en el cual dicho exceso pueda ser eliminado, constituye un caso genuino de desequilibrio. Para analizar la situación y proponer políticas adecuadas se debe, primero, tener la certeza de si el desequilibrio es por exceso de demanda o por exceso de oferta y estar en condiciones de entender las razones de la persistencia del desequilibrio y del fracaso de los mecanismos de ajuste para restaurar el equilibrio.

La existencia de desequilibrio plantea problemas ingentes no sólo para el diseño del modelo sino también para su estimación. Serán insuficientes los intentos para trazar de manera simúltanea las curvas de oferta y demanda aun cuando se sigan cuidadosamente las reglas ordinarias para la identificación del modelo. Incluso si fuera posible, los participantes en el mercado considerado podrían aún salirse de las curvas relevantes, no sólo en el sentido estocástico ordinario, sino también de manera consistente, en alguna dirección particular. Por supuesto, los problemas de estimación no son insuperables, por cierto se dispone ya de algunos modelos satisfactorios para superar estos problemas. ${ }^{4}$ La mayoría de estos modelos consisten en identificar si se trata de una situación de exceso de demanda o de exceso de oferta, sobre la base del comportamiento de los precios. Pero si el comportamiento de los precios es también estocástico y está sujeto a otras variables (como en un modelo de espectativas de precios para cambios observados en períodos previos), tal identificación puede no ser fácil, en particular si, como sucede en los PMD, las series de datos sobre precios son de mala calidad y limitadas en cantidad.

Un segundo problema que surge con la admisión de desequilibrio en cualquier mercado particular o sector de un modelo macroeconométrico, es la posibilidad de efectos secundarios o propagados a otros sectores del mercado. Supóngase que la condición de desequilibrio en el sector $A$ es por exceso de demanda ¿cuál será la reacción de los consumidores de $A$ cuyas inclinaciones de demanda se ven frustradas? Si es la de acumular activos ¿qué clase de activos? —o si es la de comprar más a otros sectores ¿en cuáles serían mayores los efectos propagadores de la demanda? ¿qué tan grandes serían? ¿qué instrumentos de política afectan la dirección así como la magnitud de la propagación?

En forma similar, si existe un exceso de oferta, tanto con relación al trabajo o aun a la producción agrícola en el sector rural jcómo reaccionan los oferentes frustrados? Pueden suprimir las compras a otros sectores, ¿pero de cuáles? ¿qué sucede con el exceso de oferta? ¿es con-

" Véase Fair y Jaffee, 1972; Fair y Kelejian, 1974; y Maddala y Nelson, 1974. 
sumida en el campo? ¿se desecha? ¿se almacena para usos futuros? ¿emigran los oferentes? ¿quiénes son los que emigran? Aunque estas preguntas se han planteado con frecuencia en la literatura sobrẻ desarrollo económico, aún no se obtienen respuestas claras. La adherencia tenaz a las formulaciones de equilibrio en toda índole de modelos tiene mucho que ver en la demora para lograr un adecuado entendimiento a este respecto.

Los efectos propagadores del desequilibrio pueden ser en extremo importantes y de gran trascendencia y aún así son ignorados del todo en modelos de equilibrio. ¿Qué sucede cuando existen varios mercados diferentes en desequilibrio? por ejemplo, por exceso de oferta de trabajo en áreas urbanas; exceso de oferta de bienes agrícolas en los sectores modernos. ¿Qué sucede con la propagación y vinculación de los efectos que resultan de la integración de estos factores? ¿se anulan o se refuerzan unos a otros? ise multiplican los efectos? Respuestas a estas preguntas sólo pueden provenir de modelos macroeconométricos que disponen de un esquema de desequilibrio general. La necesidad de tratar con interdependencia entre mercados, acentúa además el problema mencionado de estimación en la medida en que sea necesario utilizar técnicas de cálculo con ecuaciones simultáneas.

La existencia de desequilibrio plantea también cuestiones de dinámica. Por definición, el desequilibrio implica que los ajustes de precios no son instantáneos, pero ¿qué tan rápido deben ajustarse? ¿deben ajustarse las cantidades más que los precios? ¿qué tan rápido deben ocurrir los ajustes de las cantidades? ¿qué tipo de variables institucionales, políticas y de otro tipo influyen tanto en la velocidad relativa como absoluta de estos dos tipos de ajuste? Dado que los precios de equilibrio rara vez son conocidos ¿qué información hace reaccionar a oferentes y demandantes de los diferentes sectores? ¿las reacciones con respecto a las brechas entre demanda y oferta son lineales o no lineales?

Más aún, el alcance del análisis del desequilibrio en los PMD no se limita a los casos estrictos de exceso de demanda, o de oferta, en los (por lo común) relativámente pocos mercados donde los precios son fijados de manera arbitraria por agencias del gobierno. Cualquier mercado o sector en el cual los ajustes son incompletos o menos que instantáneos, está caracterizado por el desequilibrio. Este se refleja en fenómenos diversos, como tasas de natalidad elevadas en las áreas rurales, junto con una migración visible a las áreas urbanas, tecnología importada que impide el ajuste de los precios de los factores que prevalecen en los PMD y una distribución del ingreso que es menos equitativa a medida que tiene lugar el desarrollo. En verdad tales fenómenos son lo suficiente penetrantes en la mayoría de tales países como para sugerir que las formulaciones de desequilibrio deben ser centrales en los modelos para PMD más que las periféricas que se consideran en la actualidad. 


\section{Integración de las perspectivas de corto, mediano y largo plazo}

De manera típica, parece que por una parte los modelos econométricos de largo plazo, en esencia modelos de crecimiento, se basan en índices de productividad de funciones-producción muy elaboradas y con cambios en el tiempo, ${ }^{\complement}$ y en literatura por completo separada, los modelos econométricos de corto plazo enfatizan la determinación del nivel y composición de la demanda agregada. Son raros y superficiales los intentos para integrar ambos aspectos en un solo modelo macroeconométrico. Una excepción notable es el estudio de la Agencia de Planificación Económica del gobierno de Japón (1965) que contiene los siguientes modelos interrelacionados: un modelo agregado de largo plazo circunscrito al ahorro, a la formación de capital neto y a la producción potencial mediante una función-producción; un modelo sectorial de largo plazo, orientado a las diferencias en las funciones-producción y a la productividad del trabajo entre sectores, en el que se introduce el comercio exterior y se explica la asignación sectorial de recursos de trabajo y capital; un modelo de mediano plazo que trata los factores determinados en los modelos de largo plazo como variables exógenos (en particular porque fueron estimadas con series de tiempo), se determina la demanda efectiva, los precios, los salarios y la distribución del ingreso y señala los efectos de los instrumentos de política del gobierno en el sistema; y, por último, un modelo interindustrial de corto plazo que desagrega los componentes de la demanda final determinada en el modelo de mediano plazo, en las demandas de sectores individuales -externas y domésticas y calcula los requerimientos de trabajo y capital que implican tales demandas. Los modelos individuales integrados se utilizan para hacer predicciones de corto y mediano plazo. Las predicciones de cada modelo son comparadas y revisadas de manera iterativa, hasta lograr su convergencia y consistencia.

Al generalizar sobre el modelo japonés, puede sugerirse que los modelos de largo plazo pueden utilizarse también para identificar cambios de parámetros que, a su vez, identificarán varias "épocas" dentro de las cuales los parámetros de los modelos de mediano y corto plazo podrían ser constantes, aunque durante las cuales podrían cambiar todos. ${ }^{6}$ De manera inversa, los modelos de corto y mediano plazo podrían ayudar a identificar situaciones de exceso de oferta o de demanda, que podrían retroalimentar el factor de acumulación, así como otros rasgos de los modelos de largo plazo. En principio, las observaciones individuales de los modelos de largo plazo pueden ser medias aritméticas de tres, cinco, o.aun de 10 años, mientras que para los modelos de plazo

` Los ejemplos más conocidos de tales modelos son los de Solow, 1975; Denison, 1962, 1968; y Jorgenson y Griliches.

- Para una aplicación de esta técnica en un modelo de largo plazo, véase Brown, 1966. 
medio bien pudieran ser observaciones anuales $y$ semianuales o trimestrales para los de corto plazo.

Como ya se mencionó, la importancia relativa de los factores demográficos, de los cambios estructurales y tecnológicos, en el caso del crecimiento de largo plazo de los PMD, y la persistencia de diferencias dualísticas entre sectores incrementa de manera adicional los beneficios potenciales y la importancia de integrar consideraciones de corto, mediano y largo plazo en modelos macroeconométricos de los PMD. Sin tal integración, por lo general será imposible señalar las implicaciones de conjuntos de políticas macroeconómicas alternativas sobre los diversos objetivos del desarrollo.

\section{Política de planificación}

En los países desarrollados la predicción ha sido el principal uso de los modelos macroeconométricos. En realidad, a base de vender suscripciones de tales predicciones es como los diseñadores de modelos en los PD han podido financiar al menos parte del costo del diseño y de la revisión continua y actualización de sus modelos. La utilización para prueba de hipótesis e implicaciones de política con tales modelos no ha sido explotada del todo.

En los PMD, la utilización de modelos macroeconométricos para la prueba de hipótesis y deducción de implicaciones de política parece ser potencialmente de una gran importancia. Sin embargo, hasta ahora, la tendencia mencionada antes por parte de los diseñadores de modelos de los PMD que siguen las huellas de sus predecesores de los PD, y el deseo comprensible por parte de aquellos por mostrar que sus modelos proven una descripción adecuada de las economías para las que fueron diseñados, se han combinado para limitar la utilidad de los modelos de PMD en estos aspectos.

Estos defectos son artibuibles al hecho de seguir los procedimientos con respecto a la inclusión de instrumentos de política y a la manera en que son tratados. Primero, quizá porque los gobiernos en los PMD tradicionalmente han jugado un papel mucho menor en la asignación de recursos que en los $\mathrm{PD}$, la tendencia a restringir modelos de $\mathrm{PMD}$ ha conducido a una subrrepresentación desafortunada del papel de los instrumentos macroeconómicos de política. Segundo, en aquellos relativamente pocos modelos en los que se han introducido de manera suficiente instrumentos de política, el detalle es absolutamente excesivo, de tal modo que por la ausencia de un aparato para integrarlos, son pocas las conclusiones globales que pueden derivarse de ellos, o insuficientes para ser de importancia práctica para los diseñadores de las políticas, que deben tomar decisiones cuantitativas respecto a algunos impuestos específicos y tipos de gastos. En tercer lugar, con frecuencia los instru- 
mentos de política incluídos han sido tratados como variables endógenas, por ejemplo, como funciones del tiempo o del ingreso. ${ }^{7}$ Por último, la perspectiva de corto plazo de la mayoría de los modelos de PMD, puede haber disuadido a los diseñadores del modelo de incluir algunos instrumentos importantes de política, sólo porque los valores de estos han cambiado con lentitud, o a intervalos poco frecuentes, con lo cual se dificulta obtener una apreciación cuantitativa de su impacto.

Naturalmente, cuando los instrumentos de política son excluidos del todo, o incluidos sólo sobre una base ad hoc (por ejemplo, cuando convienen para propósitos de predicción), o como variables endógenas, no pueden derivarse implicaciones válidas de política.

\section{El uso de modelos internacionales de "sección transversal"}

De hecho, todas las deficiencias indicadas de los modelos macroeconométricos de los PMD están interrelacionadas, y la mayoría de ellas se pueden descubrir en el análisis final de las limitaciones de información, en particular por la brevedad del periodo para el cual se dispone de series comparables de tiempo para cualquier país en particular. La ausencia de datos explica por qué los modelos desarrollados de mayor interés a menudo son modelos de simulación. ${ }^{8}$ Sin embargo, si no están sujetos a las reglas formales de la estimación de parámetros y carecen de objetivos para evaluar lo adecuado de la formulación, los modelos de simulación - no importa lo interesantes que puedan ser- difícilmente resultan adecuados para influir en la política.

Nuestra excesiva confianza en los supuestos y los procesos de equilibrio, en gran medida es atribuible al hecho de que tales supuestos permiten eludir las interdependencias y los encadenamientos de los efectos propagadores, así como las complejidades de los procesos de ajuste parcial que aparecen en situaciones en las cuales los mercados y los procesos no están en equilibrio. La existencia de desequilibrio, como se señaló antes, genera numerosas preguntas que sólo pueden contestarse si se dispone de más y mejor información.

Así también, la preocupación por modelos macroeconométricos de PMD con consideraciones de corto plazo y el fracaso para integrar las

\footnotetext{
* Nuestra querella no es contra la endogeneización de las variables de política, pèr se. En verdad se pueden aplaudir las excursiones serias en la política económica, como las de Marzouk, 1970, en donde las variables de política son explicadas en términos del poder relativo de los diferentes grupos socio-económicos-políticos. Sin embargo, la especificación de instrumentos de política como funciones del tiempo, parece que proporciona pocos beneficios en cuanto al poder explicativo, especificación de errores, etc., relacionados con el costo del debilitamiento de la facultad de deducir del modelo conclusiones de política.

${ }^{8}$ Para algunas aplicaciones interesantes de modelos de simulación, véase Holland y Gillespie, 1963 y Shubik, 1966.
} 
de largo plazo y derivar implicaciones de política que puedan utilizar los planificadores del desarrollo, hace frente a la carencia de cambios perceptibles en algunos instrumentos importantes de política dentro del período de las observaciones de la muestra.

Modelos econométricos suplementarios, estimados sobre la base sólo de datos internacionales de "sección transversal" para PMD, o para PD y PMD combinados, puede constituir un medio valioso para obtener estimaciones de algunas interrelaciones de largo plazo, o de algunos de los elementos más elusivos del desequilibrio y sobre el impacto de los instrumentos de política que no han sido practicados en muchos PMD en el corto plazo y para los cuales existen datos. Naturalmente, tales esfuerzos sólo son suplementarios. Son bien conocidas las razones del fracaso de las estimaciones de sección transversal, manejadas necesariamente con series de tiempo, o con las relaciones "medias" obtenidas de una colección de experiencias para aplicarse a países individuales.

No obstante, el uso de estimaciones obtenidas del análisis internacional con datos de sección transversal, es lugar común en gran diversidad de aspectos del desarrollo, la mayoría de los cuales son importantes en lo que se refiere a los modelos macroeconométricos de desarrollo. Por ejemplo, los cambiosi estructurales se estiman usualmente con datos internacionales de sección transversal y por lo general se consideran válidos en el tiempo (Chenery y Taylor, 1968). De manera similar, los determinantes de las exportaciones agregadas, la composición de las exportaciones y los beneficios relativos del comercio y la ayuda, han sido estudiados de manera provechosa con datos internacionales de sección transversal (Cohen y Sisler, 1971; Chenery y Strout, 1966; Naya, 1965; De Vries, 1967; Ooms, 1966; Nugent, 1974). En diversos estudios se han estimado funciones - producción agregadas y aun sectoriales y se ha medido el impacto de la formación de capital, del crecimiento de la población, del capital humano y del comercio en un esquema contable de crecimiento con base en datos internacionales de sección transversal (Hagen y Hawrylshyn, 1969; Sommers y Suits, 1971; Kuznets, 1966; Krueger, 1968).9

En realidad, todo lo que se requiere es especificar un modelo algo más completo, que pueda integrar estos fenómenos diversos que hasta ahora han sido considerados por separado y a menudo de manera específica.

Los esfuerzos individuales en la construcción de modelos macroeconométricos generan externalidades que pueden ser capturadas, al menos parcialmente, por la cooperación entre equipos de investigadores de diferentes países y que trabajan con series de tiempo internacionales de sección transversal.

- Adelman y Morris, 1968, llegaron hasta la estimación de los parámetros de un modelo de desarrollo socio-político-económico con datos internacionales de sección transversal. 


\section{Cuadro 1}

ESPECIFICACIón de MODElos INDIVIdUALES PARA LOS PAÍSES DE AMÉrica Latina

1) $P I B=C+I+G+E x+E i-N x-N i$

2) $P N B=P I B+T T+N F Y$

3) $Y d=P N B-T e-T n-T o$

4) $C=a_{10}+a_{11} Y d+a_{12} M S-a_{13} T I M E$

5a) $K_{t}^{*}=V S_{t}^{e}$

5b) $S_{t}^{e}=(1+W) P N B_{t-1}$

5c) $I p_{t}=Z\left[K_{t}^{*}-\left(\frac{1}{1-d}\right) K_{t-1}\right]=Z V(1+W) P N B_{t-1}$ $-\frac{Z}{1+d} \mathbf{K}_{t-1}$

5) Ip $=a_{20}+a_{21} P N B_{t-1}+a_{22} K_{t-1}+a_{23} T I M E$ $+a_{24} Z c m+a_{25} C R$

5') $\quad I p=a_{20}+a_{21} P N B_{t-1}+a_{22} K_{t-1}+a_{23} Z c m+a_{24} C R$ $+a_{25} T I M E+a_{26} I p_{t-1}$

6) $E x=a_{30}+a_{31} N u s+a_{32} T e / E x+a_{33} C R+a_{34} T I M E$ $+a_{35} Z c m$

7) $E i=a_{40}+a_{41} \sum N_{j=1}^{j} i+a_{42} R^{i}+a_{43} T I M E$

8) $N x=a_{50}+a_{51} P N B+a_{52} T n / N x+a_{53} Z c m+a_{54} C R$ $+a_{58} R+a_{56} T I M E$

9) $N i=a_{60}+a_{61} P N B+a_{62} Z c m+a_{63} C R+a_{64} R$ $+a_{65}$ TIME

10) $K_{t}=\left(\frac{1}{1+d}\right) K_{t-1}+I p_{t}+I g_{t}$

11a) $T n=N x T n / N x$, por definición

11) $T n=a_{70}+a_{71} N x+a_{72} T n / N x$ 
12) $T e=a_{80}+a_{81} E x+a_{82} T e / E x$

13) $T o=a_{90}+a_{91} P I B+a_{92} T o / P I B$

14) $C R=b_{10}+b_{11} M S+b_{12} T I M E$

15) $\quad M S=b_{20}+b_{21} R M+b_{22} T I M E$

16) $B P=E x+E i-N x-N i+T T+N F Y$

17) $A G=T n+T e+T o-G-I g$

A. VARIABLES ENDÓGENAS

$\begin{array}{ll}\text { Simbolo } & \text { Variable } \\ P I B & \text { Producto interno bruto } \\ P N B & \text { Producto nacional bruto } \\ Y d & \text { Ingreso disponible } \\ C & \text { Consumo privado } \\ I & \text { Inversión total (Ip + Ig) } \\ I p & \text { Inversión privada bruta } \\ E & \text { Exportaciones totales } \\ E x & \text { Exportaciones extraregionales } \\ E i & \text { Exportaciones intraregionales } \\ N & \text { Importaciones totales } \\ N x & \text { Importaciones de fuera de la región } \\ N i & \text { Importaciones intraregionales } \\ K & \text { Acervo de capital } \\ T n & \text { Impuestos por importaciones } \\ T e & \text { Impuestos por exportaciones } \\ T o & \text { Otros impuestos netos por transferencias del gobierno } \\ & \text { al sector privado } \\ C R & \text { Acervo de crédito al sector privado } \\ M S & \text { Oferta monetaria (acervo) } \\ B P & \text { Balanza de pagos } \\ A G & \text { Ahorros gubernamentales } \\ T N E T & \text { Total de impuestos netos recibidos por transferencias } \\ & \text { al sector privado } \\ & \end{array}$


B. VARIABLES EXÓGENAS

$G$ Consumo gubernamental

Ig Inversión gubernamental

$R M \quad$ Reserva monetaria

$T n / N x \quad$ Tasa de impuestos sobre importaciones

$T e / E x \quad T a s a$ de impuesto sobre exportaciones

To/PIB "Otras" tasas de impuestos

$Z c m^{a}$

$R^{a}$

Variable Dummy por membresía en la unión aduarera

(

Variable Dummy por completar la integración carretera

TIME Tiempo (en años)

TT Ajuste por términos de comercio

NFY Ingreso neto de los factores del extranjero

Nus Indice de importaciones de Estados Unidos

POP Población

$P \quad$ Indice de precios domésticos

C. OTRos SÍmbolos

$\wedge \quad$ Sobre una variable indica estimación de dicha variaCoCR Costa Rica

E o ES El Salvador

$G \circ G U \quad$ Guatemala

$\mathrm{HoHO}$ Honduras

NoNI Nicaragua

$C M$ o $M C C A \quad$ América Central

Subíndice $t, t-1 \quad$ Período de tiempo indicado

$x \quad$ Indica valores deseados

$e \quad$ Indica valores esperados

$D \quad$ Antes de una variable (como en DRM, DMS, $D R C)$ indica primera diferencia, por ejemplo $\left(R M_{t}\right.$ - $R M_{t-1}$ ), etc.

LPI Modelo de programación lineal para un país individual (sin coordinación)

LPC Modelo de programación lineal colectivo para América Central (con coordinación) 
III. APlicación de modelos macroeconométricos a la política de Planificación Y CoORdinación: El caso de América Central

Otra justificación de suplementar modelos econométricos nacionales con gestiones internacionales, o modelos internacionales, es la interdependencia económica entre grandes socios comerciales, o países entre los cuales los recursos tienen movilidad relativamente libre. Si las economías son interdependientes, cada país es afectado indirectamente por las variables exógenas del otro país, incluidos sus instrumentos de política. Tal interdependencia ha proporcionado la motivación para las gestiones de cooperación en gran escala para la construcción de modelos macroeconométricos a nivel internacional, en especial en el proyecto LINK. Si los efectos de las políticas económicas de un país sobre otro y viceversa pueden ser determinados cuantitativamente, los países interdependientes pueden ser inducidos a coordinar sus políticas y cumplir así mejor sus metas de desarrollo.

En esta sección se describe una gestión de pequeña escala para estimar estas interdependencias en América Central, las cuales, como resultado del establecimiento de la unión aduanera conocida como.Mercado Común Centroamericano, han logrado un grado considerable de interdependencia económica. El uso potencial de modelos macroeconométricos en la política de planificación, se ejemplifica en relación con la elección de políticas óptimas para países de América Central, primero sin una política de coordinación entre países y luego con una coordinación completa de políticas. Mediante la comparación de los resultados obtenidos en estos dos casos con respecto al ingreso máximo factible, para la región en conjunto (y para los países individuales del área), se obtiene una estimación de los beneficios potenciales de la coordinación de políticas entre los países de la región.

Dado que aquí el propósito estricto es ilustrar el uso posible de modelos econométricos para políticas de planificación y coordinación, el modelo en sí, su presentación y los métodos utilizados, son simples y concisos en la medida de lo posible. Al lector interesado en detalles se recomienda otras fuentes (Nugent, 1974). Como puede observarse en el cuadro 1, el modelo utilizado es de un tipo keynesiano relativamente "ingenuo", y por lo tanto su utilidad se limita enteramente al corto plazo. Los modelos utilizados consisten en modelos macroeconométricos separados de cada país de América Central, compuestos de 17 ecuaciones con 17 variables endógenas especificadas de manera uniforme para todos los países. Los cinco modelos se interrelacionan a través de las variables de exportaciones interregionales de cada país $i\left(E i^{i}\right)$ que están influidas por el nivel de importaciones interregionales de cada uno de los otros países $j$ de la región $\left(\mathrm{Ni}^{i}\right)$. Existen seis instrumentos de política en cada país: el consumo del gobierno $(G)$; la inversión del gobierno (Ig); la reserva monetaria (RM); y las tasas de impuestos sobre las im- 
portaciones $(T n / N x)$, sobre las exportaciones $(T e / E x)$ y sobre el ingreso $(T o / P I B)$.

Las soluciones de las ecuaciones estructurales para cada país se obtuvieron mediante dos estimaciones de mínimos cuadrados. Los resultados obtenidos para Costa Rica y El Salvador se presentan como ejemplo en los cuadros $2 \mathrm{~A}$ y $2 \mathrm{~B}$.

En virtud de que la inversión está directamente afectada por los retardos de las variables endógenas, y por lo cual casi todas las variables endógenas están afectadas de manera indirecta por tales variables, el modelo permite analizar los efectos de los instrumentos de política no sólo sobre las variables del período corriente, sino también sobre las de períodos futuros. Puesto que en la formación del modelo no fueron utilizados retardos de longitud mayor a un año, la mayoría de estas interconecciones dinámicas entre instrumentos de política y otras variables, empezarán a percibirse hasta después de dos años. Por esta razón, y para presentar el modelo de la manera más simple posible y no extenderlo más allá de las capacidades permisibles de corto plazo, se expandió el modelo de modo de incluir sólo dos periodos. (años). Esto duplica el número de ecuaciones, 34 por país (excepto para Costa Rica y Honduras, que carecen de ecuaciones para el crédito). Estos sistemas de 34 ecuaciones por país pueden tratarse en forma alternativa como modelos separados o pueden manejarse en forma conjunta, como un gran modelo para América Central, con 170 ( 34 x 5) ecuaciones. Al agregarse las identidades siguientes para cada uno de los dos períodos, para América Central en conjunto, el sistema completo consiste de 178 ecuaciones.

$$
\begin{aligned}
& P I B^{M C C A}=P I B^{C R}+P I B^{E S}+P I B^{G U}+P I B^{H O}+P I B^{N I} \\
& C^{M C O A}=C^{C R}+C^{E S}+C^{O U}+C^{H O}+C^{N I} \\
& I^{H O C A}=I^{C R}+I^{E S}+I^{Q U}+I^{H O}+I^{N I} \\
& \quad \sum_{j=1}^{5} N i^{j}=\sum_{i=1}^{5} E i^{j}+\text { costo de transporte entre países } \\
& \text { para cada año } t \text { y } t+1
\end{aligned}
$$

Este sistema de 178 ecuaciones es de la forma:

(a) $I Y=A Y+B X$

Donde $Y$ es el vector de 178 variables endógenas y $X$ es el vector de 98 variables predeterminadas y un término constante. $A$ y $B$ son matrices de $178 \times 178$ y $178 \times 99$, respectivamente. La solución a este sistema está dada por:

(b) $Y=(1-A)^{-1} B X$

Este sistema de ecuaciones puede considerarse la forma reducida del modelo, dado que cada una de las 178 variables endógenas contenidas en $Y$ se expresa como una función lineal solamente de las 99 variables 
Cuadro $2 \mathrm{~A}$

Modelo 1: Estimaciones para Costa Rica

\begin{tabular}{|c|c|c|c|}
\hline \multicolumn{2}{|c|}{ Fouraion } & \multirow{2}{*}{$\frac{\mathrm{R}^{2}}{0.9793}$} & \multirow{2}{*}{$\frac{\mathrm{Din}}{0.60}$} \\
\hline 15). & 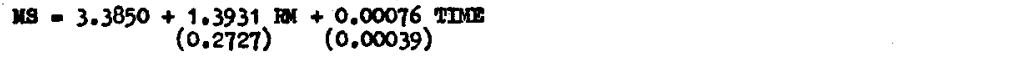 & & \\
\hline 14) & b/ & & \\
\hline 5) & 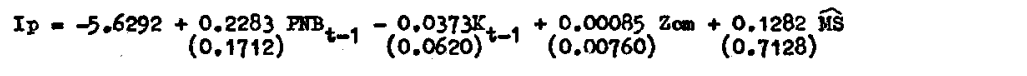 & 0.8066 & 2.77 \\
\hline 6) & 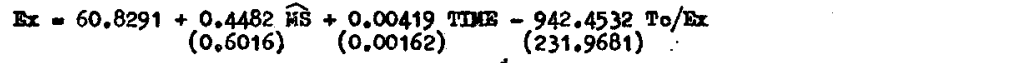 & 0.9347 & 2.89 \\
\hline 7) & 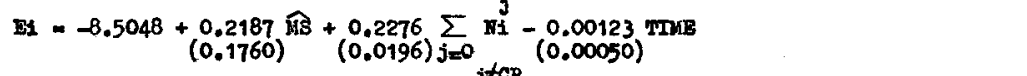 & 0.9514 & 1.44 \\
\hline 12) & 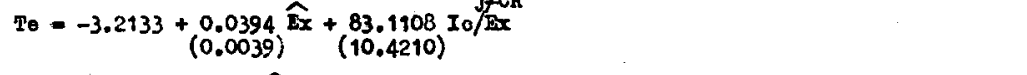 & 0.9452 & 1.83 \\
\hline 4) & $c=2.5152+0.0783 \widehat{x d}$ & 0.9978 & 1.98 \\
\hline 8) & 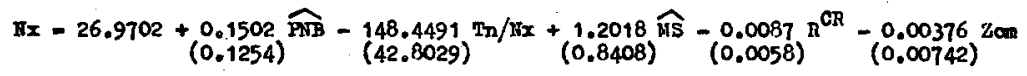 & 0.9361 & 2.23 \\
\hline 9) & 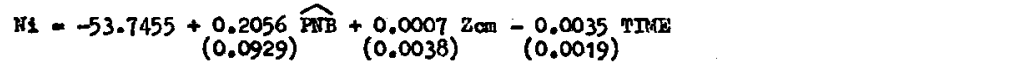 & 0.7158 & 1.25 \\
\hline 11) & $\mathrm{Tn}_{\mathrm{n}}=-28.8671+\underset{(0.0161)}{0.2655 \widehat{\mathrm{Nx}}}+\frac{111.3581 \mathrm{In} / \mathrm{Nx}}{(6.1096)}$ & 0.9852 & 2.17 \\
\hline 13) & $\begin{array}{c}\text { To }_{0}=-31.23+0.0700 \mathrm{PIB}+446.9741 \mathrm{TO} / \mathrm{PIB} \\
(0.0027) \quad(45.3244)\end{array}$ & 0.9847 & 1.13 \\
\hline
\end{tabular}

- Prueba Durwin Watson.

- No se estimó una ecuación sobre el crédito porque no se disponía de una serie consistente sobre el crédito al sector privado. Se utilizó $M S$ en lugar de $C R$ de otra sección del modelo. 
Modelo 1: Estimaciones para El Salvador

\begin{tabular}{|c|c|c|c|}
\hline \multicolumn{2}{|c|}{ Eouación } & \multirow{2}{*}{$\begin{array}{l}\bar{B}^{2} \\
0.7656\end{array}$} & \multirow{2}{*}{$\frac{\mathrm{DW}}{0.56}$} \\
\hline 15) & us $=32.2528+\frac{0.8167}{(0.1155)}$ & & \\
\hline 14) & $\mathrm{CR}=-242.5528+4.2036 \widehat{\mathrm{MS}}$ & 0.6000 & 0.40 \\
\hline 5) & $\left.I_{p}=-18.7532+\frac{0.08424}{(0.04006)}\right)_{t-1}+\frac{0.2398}{(0.1118)} \widehat{C R}$ & 0.8260 & 2.62 \\
\hline 6) & 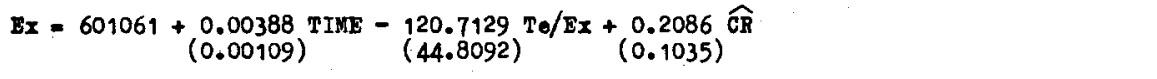 & 0.9517 & 1.82 \\
\hline 7) & 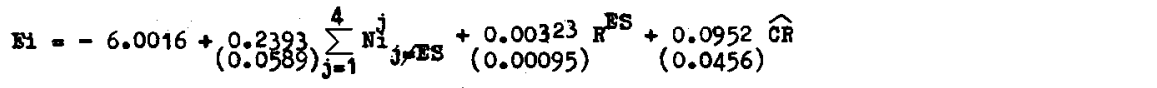 & 0.9893 & 1.87 \\
\hline 12) & 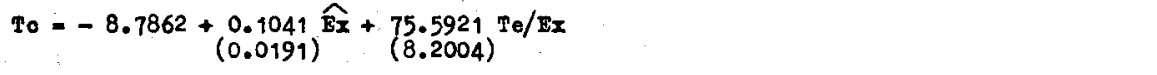 & 0.8806 & 1.42 \\
\hline 4) & $c=-24.5543+(0.9482 \widehat{y d}$ & 0.9859 & 2.28 \\
\hline 8) & 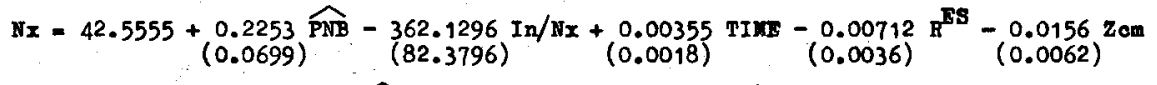 & 0.9704 & 2.90 \\
\hline 9) & 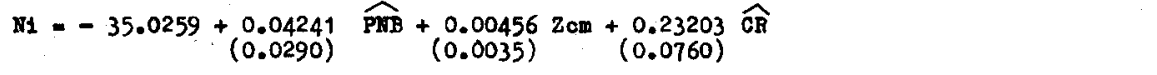 & 0.9362 & 2.23 \\
\hline 11) & 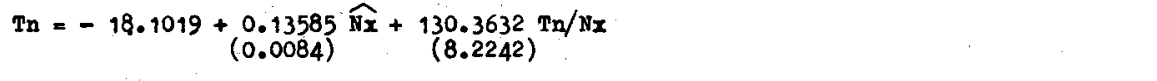 & 0.9518 & 2.58 \\
\hline 13) & 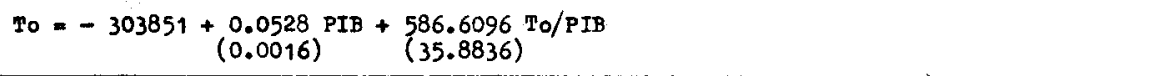 & 0.9944 & 2.00 \\
\hline
\end{tabular}

Prueba Durwin Watson. 
predeterminadas (el término constante se considera como una "variable" cuyo valor se fija en 1.0 ).

Cuando los cálculos requeridos se efectúan al margen de las matrices $A$ y $B$ en cualquiera de los tres modelos, cuyos resultados se expusieron antes, se obtiene un conjunto de $176 \times 99$ coeficientes de la forma reducida. Tal matriz de coeficientes expresa los impactos, en términos cuantitativos, de cada una de las 99 variables determinadas, sobre cada una de las 178 variables endógenas. Dado que muchas de las variables endógenas son de un interés secundario y que algunas de las variables predeterminadas no constituyen instrumentos de política, no es necesario considerar la matriz completa de coeficientes en su forma reducida.

En lugar de lo anterior, centramos la atención en los coeficientes, en su forma reducida, de cada uno de los seis instrumentos de política, $G, I g, R M, \frac{T n}{N x}, \frac{T e}{E x}, \mathrm{y} \frac{T o}{P I B}$, acerca de cinco de las variables objetivo más importantes, o sea el $P N B$, el consumo privado $(C)$, la inversión $(I)$, los ahorros del gobierno $(A G)$, y la balanza de pagos $(B P)$. Como ejemplo de estos resultados, en el cuadro 3 se presenta el conjunto de coeficientes, en su forma reducida, que representan el impacto de un cambio de $1 \%$ en el ingreso y las tasas de impuesto a las ventas (considerados en el modelo como "otros" impuestos) sobre tres de las variables objetivo ( $P N B, A G$ y $B P$ ) en cada país y para cada uno de los dos períodos de tiempo $t$ y $t_{1}+1$.

Aunque el impacto primario de los cambios en los instrumentos de política se percibe en los países en que se toma la acción, de estos resultados podría derivarse que los cambios de política tienen efectos sustanciales en otros países del MCCA. No sorprende encontrar que la difusión de los efectos sobre los otros países tienda a ser mayor en el caso de Honduras y Nicaragua, países que registran mayor déficit de comercio intraregional. Otra característica que se deriva de estos resultados del cuadro 3, es que el impacto de estos cambios de políticas no se restringe a un solo período de tiempo. En realidad, en diversas instancias, los efectos retardados de los cambios de política son tan grandes o aún mayores que los efectos inmediatos.

El trabajo de análisis económico se completa con estas estimaciones de los efectos de los instrumentos de política y otras variables indeterminadas sobre las variables objetivo en el tiempo (año $t$ y $t+1$ ) y en el espacio (en cada uno de los países de América Central). ${ }^{10}$ De este análisis se obtiene una matriz $M$ por cada país, variable, y multiplicadores específicos fechados de los vectores, $X_{A}$, de instrumentos de política sobre el vector de variables endógenas $Y$ que interesan, $Y_{A}$, o sea sobre las

${ }^{10}$ Al multiplicar los valores de estas otras variables predeterminadas por los coeficientes relevantes en su forma reducida, estos efectos pueden considerarse por separado y consolidarse en el término constante. 
Efectos sobre El $P I B$ de Un aumento de $1 \%$ EN LA TASA DE "OTros impuestos" (EN millones de pesos de América Central)

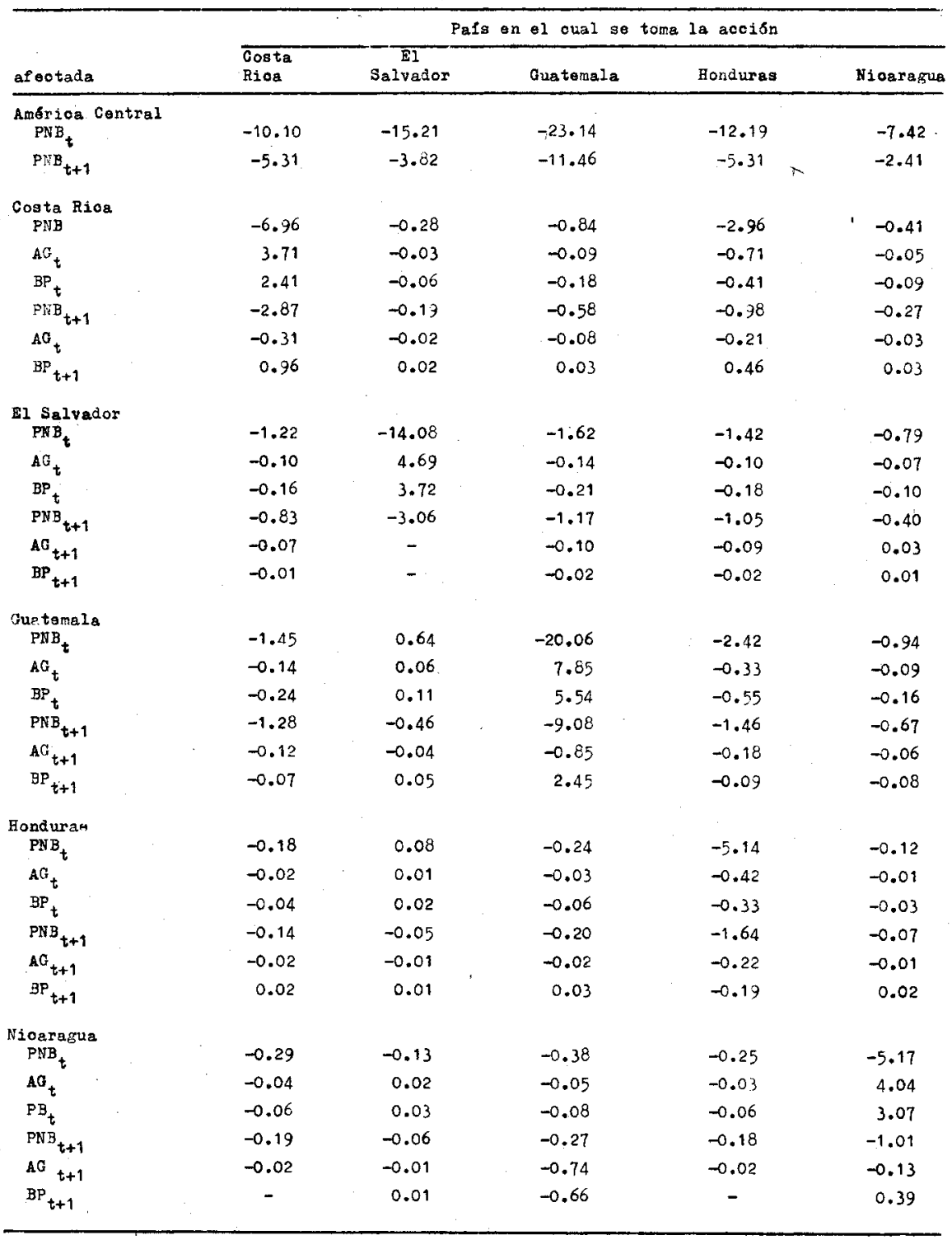


cinco variables objetivo: $P N B, C, I, A G$ y $B P, M$ es entonces la parte importante de la matriz $(I-A)^{-1} B$ completa en su formà reducida.

Podemos pasar ahora al análisis de política, en donde se invierte la dirección del análisis, o sea, la determinación de los instrumentos de política óptimos en términos de las metas establecidas. En lugar de metas fijas, se supone ahora que éstas son flexibles y que pueden ser establecidas en términos de maximización y de restricciones de desigualdad.

En primer lugar se elige específicamente una de las metas en $Y_{A}$, por ejemplo el $P I B$ como la única meta flexible por maximizar:

$$
P N B_{t}+P N B_{t+1}=\text { Máximo }
$$

En segundo lugar, para cada año, $t$ y $t+1$ se establece un conjunto adecuado de restricciones sobre cada una de las otras variables objetivo: consumo $(C)$, inversión $(I)$, balanza de pagos $(B P)$ y los ahorros del gobierno $(A G)$. Específicamente, los valores elegidos para las restricciones fueron valores reales de estas variables, para años recientes consecutivos para los cuales se disponía de información (1967 y 1968).

$$
\begin{gathered}
C \geqq C \text { Min } \\
I \geqq I \text { Min } \\
B P \\
\geqq B P \text { Min } \\
A G \geqq A G \text { Min }
\end{gathered}
$$

En seguida se incluyen las alternativas $(M)$ entre los instrumentos $\left(X_{A}\right)$ y las cinco variables objetivo $\left(Y_{A}\right)$, con lo cual se constituyen otras cinco ecuaciones ( 6 a 10 ) para cada uno de los dos años.

Por último, se establecen límites superiores e inferiores para cada uno de los instrumentos de política para representar las restricciones de política sobre los cambios límite de las mismas. Los límites superior e inferior elegidos reflejan los cambios máximos positivos y negativos de un año a otro, o sea las desviaciones de la tendencia histórica- el que parezca más apropiado en el país particular considerado (Nugent, 1974, cuadros 5-1 y 5-2).

$$
\begin{aligned}
& \frac{T e}{E x} \geqq \frac{T e}{E x} \text { Min } \\
& \frac{T n}{N x} \geqq \frac{T n}{N x} \text { Min } \\
& \frac{T o}{P I B} \geqq \frac{T o}{P I B} \text { Min }
\end{aligned}
$$




$$
\begin{aligned}
R M & \geqq R M \text { Min } \\
G & \geqq G \text { Min } \\
I g & \geqq I g \text { Min } \\
\frac{T e}{E x} & \leqq \frac{T e}{E x} \operatorname{Max} \\
\frac{T n}{N x} & \leqq \frac{T n}{N x} \mathrm{Max} \\
\frac{T o}{P I B} & \leqq \frac{T o}{P I B} \operatorname{Max} \\
R M & \leqq R M \operatorname{Max} \\
G & \leqq G \operatorname{Max} \\
I g & \leqq I g \operatorname{Max}
\end{aligned}
$$

Dado que se pueden establecer escalas para todas las variables ( $y$ consecuentemente modificarse las restricciones y las ecuaciones), se pueden agregar restricciones no negativas sobre cada una de las variables objetivo y de las variables instrumentales. Esto lleva a un sistema de ecuaciones y desigualdades ( 1 a 22 ) del tipo de un modelo de programación lineal:

$$
\begin{array}{cl}
\text { Max } & C X \\
(I-A) & X \leqq B \\
& X \supseteqq 0
\end{array}
$$

El "dual" de la formulación del anterior "primario" de programación lineal, es de la forma:

$$
\begin{array}{r}
\text { Min } P B^{\prime} \\
P[I-A]^{\prime} \geqq C^{\prime} \\
P \geqq 0
\end{array}
$$

La forma de programación lineal ofrece la gran ventaja de que las soluciones, tanto para el "primario" como para el "dual", pueden obtenerse de manera rápida y fácil. Si existe una solución factible tanto para el "primario" como para el "dual", por lo general existe un número 
infinito de soluciones entre las cuales puede elegirse la solución óptima del "primario". De tal solución puede obtenerse un conjunto de políticas óptimas $\left(X_{A}^{*}\right)$ así como el máximo bienestar factible $\left(C X_{A}^{*}\right)$, dadas ciertas restricciones. De la solución dual se obtienen los precios sombra óptimos $(p)$ que representan el costo, en términos de bienestar de reducir cada restricción particular en una unidad. En este caso los precios sombra proveen: 1) las alternativas de cada limitación sobre cada instrumento de política y el criterio de bienestar $(P N B)$ y; 2) las alternativas entre cada una de las otras metas de bienestar $(C, I, B P$, y $A G)$ y el $P N B$. Dado que todos los precios sombra constituyen precios relativos y que todos son porcientos respecto a la misma base (en este caso el $P N B$ ), las tasas de los diferentes precios sombra de las diferentes restricciones pueden también calcularse, por lo que una matriz global de todos los posibles costos alternativos, entre cada instrumento de política y las metas de bienestar, pueden obtenerse de manera fácil. Estos costos alternativos son los diversos costos posibles. Con el conocimiento de estas posibilidades de costos alternativos, el diseñador de política puede elegir la óptima igualando los posibles costos alternativos con los deseables. ${ }^{11}$

Nuestro objetivo presente se concentra sólo en la solución del "primario". De manera específica se desea comparar el bienestar susceptible de obtenerse cuando es posible la coordinación completa de políticas entre países de la región (al utilizar el modelo global de Centroamérica) con el bienestar susceptible cuando no es posible la coordinación de políticas entre los miembros del MCCA (al utilizar modelos nacionales separados). Por lo tanto, concentraremos nuestra atención en las soluciones del problema "primario", los valores óptimos de los instrumentos de política y en especial de las variables objetivo.

Al utilizar el modelo de la manera descrita para América Central en conjunto, del cuadro 4, se obtienen los valores para 1967 y 1968 (en las primeras dos columnas, o sea las señaladas con "LPC no ajustado") a partir de la solución primaria óptima del modelo de programación lineal. Se observa que en todos los casos los valores del PNB y de casi. todas las otras variables objetivo, son mayores en el modelo de América Central (en el cual las políticas de todos los países se determinan de manera simultánea) que en los modelos de los países considerados aisladamente (en los cuales las políticas en cada uno de ellos se determinan también por separado).

Sin embargo, la diferencia entre estos dos conjuntos de valores de soluciones óptimas, no es atribuible sólo a los diferentes enfoques de deter-

\footnotetext{
${ }^{11}$ Este método fue desarrollado y aplicado en un contexto algo diferente, en Nugent y DePrano, 1966. Un problema general se deriva de que este procedimiento se basa en un análisis aditivo y pertenece a periodos de tiempo discretos. Esto limita la capacidad del modelo para lograr mucho en el sentido de una "sintonización" precisa de la elección de políticas. Por esta razón muchos analistas están utilizando enfoques de teorías del control para la elección de políticas (Intriligator, 1971).
} 
minación de política, sino también a los errores de estimación de las ecuaciones de importaciones interrregionales, $(\mathrm{Ni})$-las únicas que han sido tratadas de manera diferencial en el modelo agregado de América Central y en los modelos de países individuales. Esto va en contra del hecho de que las variables $\mathrm{Ni}$ de los otros países son consideradas como exógenas en los modelos de países individuales - que por supuesto son consideradas endógenas en el modelo agregado de América Central. En el primer conjunto de modelos no se involucran modelos de estimación, mientras que en el último se introducen modelos de estimación de errores. Para tomar en cuenta esta diferencia en el tratamiento, se estimó la diferencia entre los valores de los términos constantes en las ecuaciones consolidadas y entre las ecuaciones abreviadas de forma reducida atribuibles a esta fuente. De esta manera se han ajustado incrementando los términos constantes originales de los países individuales en el grado en que los modelos de América Central sobreestimaban cada variable endógena y, disminuyéndolos en la medida en que los modelos subestimaban cada variable endógena. Por el contrario, el conjunto opuesto de ajustes se puede hacer en los términos constantes del modelo de América Central, para hacer comparables los resultados con las soluciones no ajustadas de los países individuales. Las soluciones óptimas obtenidas de los modelos individuales ajustados, aparecen ahora en el segundo par de columnas (las indicadas por "LPI ajustado") en cada uno de los cuadros. De manera similar, las soluciones óptimas que se obtienen del modelo ajustado de América Central, aparecen en el quinto par de columnas en cada uno de estos cuadros (aquellas indicadas por "LPC ajustado").

Los valores de la solución para las variables objetivo, del primero y segundo pares de columnas, son comparables ahora en la medida en que se considera el error de estimación en las ecuaciones Ni. De manera similar, y por las mismas razones, el cuarto y quinto par de columnas son comparables. Como puede observarse fácilmente, sin excepción, cuando es posible la coordinación completa de políticas entre países (como en el modelo de América Central) es posible alcanzar objetivos mayores que cuando tal coordinación no existe.

Sin embargo, es probable que nuestras comparaciones sobreestimen los verdaderos beneficios de la coordinación regional de políticas macroeconómicas en América Central. Esto es así dado que las optimizaciones en los países individuales se computaron sin la ventaja del conocimiento acerca de las decisiones óptimas de política en los otros países, mientras que la coordinación completa de políticas, en el modelo de América Central, permitió disponer de información completa. Podría preguntarse cuál sería la mejor manera de que los países individuales tuvieran éxito, con respecto a sus objetivos, de haber existido información completa sobre lo que hicieron los otros países acerca de sus políticas óptimas. Se ha hecho una reestimación de las maximizaciones con el modelo de los países aislados, con el supuesto de que cada país individual conocía las 
Cuadro 4

SOLUCIONES ÓPTIMAS

\begin{tabular}{|c|c|c|c|c|c|c|c|c|c|c|}
\hline \multirow{3}{*}{ '. } & \multicolumn{6}{|c|}{ Comparaeion 1} & \multicolumn{4}{|c|}{ Comparaoion 2} \\
\hline & \multicolumn{2}{|c|}{ LPC no s justado } & \multicolumn{2}{|c|}{ LPI a justado } & \multicolumn{2}{|c|}{$\begin{array}{l}\text { LPI a jus tado } \\
\text { J oon informa } \\
\text { oibn oompletata }\end{array}$} & \multicolumn{2}{|c|}{ LPI no a justado } & \multicolumn{2}{|c|}{ LPC ajustade } \\
\hline & 1967 & 1968 & 1967 & 1968 & 1967 & 1968 & 1967 & 1968 & 1967 & 1968 \\
\hline $\begin{array}{l}P N B-C R \\
C C-C R \\
I p-C R \\
A G-C R \\
B P-C R\end{array}$ & $\begin{array}{l}795.0 \\
594.2 \\
100.1 \\
-32.9 \\
-43.4\end{array}$ & $\begin{array}{r}853.2 \\
615.0 \\
175.2 \\
8.5 \\
-43.4\end{array}$ & $\begin{array}{l}709.7 \\
508.7 \\
100.1 \\
-20.9 \\
-43.4\end{array}$ & $\begin{array}{r}763.8 \\
547.2 \\
124.8 \\
-1.3 \\
-43.4\end{array}$ & $\begin{array}{l}686.0 \\
491.7 \\
100.1 \\
-18.8 \\
-43.4\end{array}$ & $\begin{array}{r}779.5 \\
559.4 \\
119.6 \\
3.1 \\
-43.4\end{array}$ & $\begin{array}{l}725.3 \\
524.3 \\
100.1 \\
-23.1 \\
-43.4\end{array}$ & $\begin{array}{r}780.2 \\
557.9 \\
128.3 \\
0.5 \\
-43.4\end{array}$ & $\begin{array}{l}811.3 \\
595.6 \\
100.1 \\
-35.0 \\
-43.4\end{array}$ & $\begin{array}{r}871.2 \\
627.4 \\
178.9 \\
10.2 \\
-43.4\end{array}$ \\
\hline $\begin{array}{l}\text { PNQ-ES } \\
C-\mathbb{E S} \\
\mathrm{IP}_{\mathrm{PSS}} \\
\mathrm{AQ} \mathrm{ESS} \\
\mathrm{BP}-\mathrm{ES}\end{array}$ & $\begin{array}{r}1089.9 \\
899.1 \\
119.0 \\
5.6 \\
-38.5\end{array}$ & $\begin{array}{r}1169.4 \\
961.0 \\
155.3 \\
38.5 \\
-38.5\end{array}$ & $\begin{array}{r}976.3 \\
805.3 \\
119.0 \\
11.7 \\
-38.5\end{array}$ & $\begin{array}{r}1007.0 \\
820.7 \\
135.3 \\
26.1 \\
-38.5\end{array}$ & $\begin{array}{r}985.0 \\
824.7 \\
119.0 \\
10.7 \\
-38.5\end{array}$ & $\begin{array}{r}1049.5 \\
847.8 \\
141.3 \\
30.1 \\
-38.5\end{array}$ & $\begin{array}{r}1025.5 \\
854.5 \\
119.0 \\
8.0 \\
-38.5\end{array}$ & $\begin{array}{r}1067.3 \\
872.8 \\
143.6 \\
31.5 \\
-38.5\end{array}$ & $\begin{array}{r}1144.0 \\
953.3 \\
119.0 \\
1.5 \\
-38.5\end{array}$ & $\begin{array}{r}1184.0 \\
1012.3 \\
157.1 \\
40.2 \\
-38.5\end{array}$ \\
\hline $\begin{array}{l}P N B-G \\
Q-G \\
I p-G \\
A G-G \\
B P-G\end{array}$ & $\begin{array}{rr}1 & 667.6 \\
1 & 366.3 \\
141.1 \\
-36.7 \\
-52.8\end{array}$ & $\begin{array}{r}1917.6 \\
1584.3 \\
193.3 \\
-13.9 \\
-65.8\end{array}$ & $\begin{array}{rr}1 & 551.9 \\
1 & 260.0 \\
141.1 \\
-36.7 \\
-62.4\end{array}$ & $\begin{array}{r}1742.6 \\
1441.0 \\
170.4 \\
-27.1 \\
-65.8\end{array}$ & $\begin{array}{r}1585.8 \\
1290.2 \\
141.1 \\
-36.7 \\
-59.6\end{array}$ & $\begin{array}{r}1759.6 \\
1455.1 \\
177.1 \\
-21.7 \\
-65.8\end{array}$ & $\begin{array}{r}1594.1 \\
1298.8 \\
141.1 \\
-36.7 \\
-58.9\end{array}$ & $\begin{array}{r}1808.3 \\
1494.8 \\
178.8 \\
-23.6 \\
-65.8\end{array}$ & $\begin{array}{r}1714.6 \\
1.409 .4 \\
141.1 \\
-36.7 \\
-49.0\end{array}$ & $\begin{array}{r}1 \quad 985.8 \\
1607.2 \\
195.9 \\
-9.9 \\
-65.8\end{array}$ \\
\hline $\begin{array}{l}\text { PNB-H } \\
C-H \\
I_{p}-H \\
A G-H \\
B P-H\end{array}$ & $\begin{array}{r}654.2 \\
486.9 \\
65.1 \\
-17.3 \\
-3.3\end{array}$ & $\begin{array}{r}701.2 \\
521.8 \\
101.6 \\
-9.2 \\
-16.4\end{array}$ & $\begin{array}{r}627.6 \\
466.9 \\
65.1 \\
-17.3 \\
-6.5\end{array}$ & $\begin{array}{r}665.9 \\
496.2 \\
83.2 \\
-13.8 \\
-16.4\end{array}$ & $\begin{array}{r}630.0 \\
467.7 \\
65.1 \\
-17.3 \\
-6.2\end{array}$ & $\begin{array}{r}669.8 \\
499.2 \\
84.0 \\
-13.5 \\
-16.4\end{array}$ & $\begin{array}{r}647.4 \\
481.1 \\
65.1 \\
-17.3 \\
-4.2\end{array}$ & $\begin{array}{r}689.0 \\
512.6 \\
89.7 \\
-10.0 \\
-16.4\end{array}$ & $\begin{array}{r}673.9 \\
504.0 \\
65.1 \\
-17.3 \\
-0.5\end{array}$ & $\begin{array}{r}724.8 \\
538.8 \\
108.0 \\
-5.5 \\
-16.4\end{array}$ \\
\hline $\begin{array}{l}P N B-N \\
C-N \\
I p-N \\
A G-N \\
B P-N\end{array}$ & $\begin{array}{r}624.5 \\
460.9 \\
92.3 \\
-25.2 \\
-35.7\end{array}$ & $\begin{array}{l}709.5 \\
526.5 \\
126.0 \\
-25.2 \\
-45.4\end{array}$ & $\begin{array}{r}603.7 \\
437.8 \\
-32.3 \\
-25.2 \\
-38.4\end{array}$ & $\begin{array}{l}677.4 \\
497.8 \\
127.8 \\
-25.2 \\
-45.0\end{array}$ & $\begin{array}{r}614.2 \\
447.1 \\
92.3 \\
-25.2 \\
-37.3\end{array}$ & $\begin{array}{l}690.4 \\
508.7 \\
129.4 \\
-25.2 \\
-45.3\end{array}$ & $\begin{array}{r}606.5 \\
440.3 \\
92.3 \\
-25.2 \\
-38.1\end{array}$ & $\begin{array}{l}681.4 \\
501.4 \\
116.5 \\
-25.2 \\
-45.0\end{array}$ & $\begin{array}{r}629.0 \\
460.4 \\
92.3 \\
-25.2 \\
-35.7\end{array}$ & $\begin{array}{l}713.9 \\
530.4 \\
141.2 \\
-25.2 \\
-45.1\end{array}$ \\
\hline $\begin{array}{l}\mathrm{PNB}-\mathrm{CA} \\
\mathrm{C}-\mathrm{CA}^{-}\end{array}$ & $\begin{array}{l}4831.2 \\
3807.4\end{array}$ & $\begin{array}{l}5351.0 \\
4172.6\end{array}$ & $\begin{array}{ll}4 & 469.2 \\
3 & 478.7\end{array}$ & $\begin{array}{ll}4 & 856.7 \\
3 & 802.9\end{array}$ & $\begin{array}{l}4595.2 \\
3596.7\end{array}$ & $\begin{array}{l}4948.8 \\
3901.5\end{array}$ & $\begin{array}{l}4598.8 \\
3599.0\end{array}$ & $\begin{array}{l}5026.2 \\
3939.5\end{array}$ & $\begin{array}{l}4972.9 \\
3922.6\end{array}$ & $\begin{array}{ll}5 & 524.2 \\
4 & 315.8\end{array}$ \\
\hline
\end{tabular}


soluciones óptimas de los otros, insertando valores óptimos de $\sum_{j=1}^{4} N i_{j}^{j} \neq 1$ (en lugar de valores reales) en los coeficientes en su forma reducida, para llegar a los valores de los términos constantes en su forma reducida y consolidada abreviada. Los términos constantes que resultan pueden ser considerados aparte (sin ajustarse) o ajustarse para tomar en cuenta el error de estimación en los términos $N i$, como se hizo antes. Este conjunto de soluciones óptimas para los modelos de los países individuales, que resultan de la última operación, se muestran en el tercer par de columnas (indicados con "LPI información ajustada y completa").

El lector puede observar con facilidad que la provisión de información completa por lo general aporta los valores de las variables objetivo de los modelos de política no coordinada (los modelos de países individuales) algo parecidos a los obtenidos de "LPC no ajustado", del modelo completo de América Central con coordinación completa de política. Sin embargo, el efecto de información completa por lo general no es muy grande - se considera sólo para una pequeña fracción de las diferencias entre las soluciones comparables obtenidas en condiciones de coordinación completa de políticas y en ausencia de tal coordinación.

Para probar la sensibilidad de los resultados ante cambios en los supuestos, en los valores de los parámetros y en las restricciones de política, se repite el mismo procedimiento para dos especificaciones diferentes de los modelos macroeconométricos, así como para conjuntos alternativos de límites sobre la flexibilidad de instrumentos de política y objetivos secundarios. Los resultados obtenidos en estas situaciones alternativas, como se registran en Nugent (1974), indican que los beneficios potenciales de la coordinación de políticas entre países de América Central, oscila de 2 a $7 \%$ del $P N B$ de la región. El hecho de que los resultados fueran por lo general poco insensibles a cualquiera y a todos los cambios, y que los modelos subyacentes permitieran en apariencia efectuar de manera satisfactoria las predicciones y retrovisiones, permite esperar que los hallazgos, bastante sustanciales, de los beneficios potenciales de la coordinación de políticas pueden ser muy considerables. Dada la distribución de las ganancias potenciales entre países, los resultados indican que los países pequeños, como Costa Rica y El Salvador, por lo general se benefician más que los grandes, y que los ricos se benefician más que los pobres, como Honduras.

Los altos ingresos son atribuibles al hecho de que, con coordinación, cada país individual esta más capacitado para adoptar políticas monetarias, expansionistas y fiscales que en ausencia de coordinación. Por ejemplo, la coordinación hace posible para Costa Rica proseguir políticas expansionarias de gasto (mayor $G$ e $I g$ ) y reducir sus tasas de impuesto a la exportación y otros impuestos $(T e / E x$ y $T o / P I B$, respectivamente), para regresar a políticas monetarias mucho menos expansionarias $(R M)$. El Salvador está en posibilidad de incrementar el gasto 
gubernamental en el período corriente ( $t$ ) y la oferta monetaria en el año $t+1$ al costo de una relación sustancialmente mayor entre otros impuestos, $T o / P I B$. De manera similar, Guatemala está en posibilidad de obtener un incremento alternativo en la tasa de "otros impuestos" en el año $t+1$ por una reducción de la tasa de otros impuestos en el año $t$ y un aumento en el gasto del gobierno en el año $t+1$. Honduras puede reducir la tasa de "otros impuestos" en el año $t$ y aumentar el gasto del gobierno en el año $t+1$. Por último, Nicaragua puede reducir la tasa de "otros impuestos" en el año $t$ y bajar la tasa de impuestos a la exportación en el año $t+1$.

Estas soluciones reflejan que la mayor flexibilidad que se deriva de una solución simultánea para todos los países, permite eliminar debilidades respecto a algunos de los recursos menos comprometidos o algunas restricciones por la flexibilidad adicional de éstos.

Sin embargo, todas las conclusiones anteriores deben considerarse tentativas en tanto puedan ser corroboradas a través de otras investigaciones, con mayor disponibilidad de información y modelos más satisfactorios.

IV. Algunas SUgerencias para la institucionalización DE LA INVESTIGACIÓN SOBRE MODELOS MACROECONÓMICOS EN PAÍSES DE ESCASO DESARROLLO RELATIVO

Mi propia experiencia en el diseño; algo primitivo, se admite, de modelos macroeconómicos para propósitos de política de planiflcación, me ha conducido a las siguientes sugerencias sobre como debe organizarse la investigación de este tópico si se desea tener éxito. En primer lugar, la construcción de un modelo macroeconométrico debe ser un proceso dinámico. Es presumible que por la falta de financiamiento, muchos esfuerzos de construcción de modelos en países de escaso desarrollo hayan sido esporádicos y discontinuos. Es alentador encontrar que un modelo para un país de América Latina (Beltran del Río y Klein, 1973) ha llegado a cinco versiones en un período de cuando mucho 5 años y no debe sorprender que los resultados hayan sido superiores, cuantitativa y cualitativamente, que para la mayoría de los modelos de otros países en desarrollo. Debería disponerse de más resultados sobre esta clase de investigaciones a nivel nacional, regional e internacional.

En segundo lugar, es muy difícil para cualquier investigador o equipo de investigadores que construyen modelos, ser completamente "objetivo" en su formulación y por lo tanto hacer justicia a todas las situaciones políticas. Por lo tanto, para demostrar los beneficios y costos alternativos de conjuntos de política y estrategias de desarrollo, debe hacerse un gran esfuerzo para fomentar la competencia entre diferentes equipos de investigadores, cada uno con su propio modelo y "visión" de la eco- 
nomía, aunque todos con la misma información y probablemente con los mismos procedimientos de estimación.

Por último, debe constituirse un consorcio de investigadores en diferentes países en tanto las diferentes experiencias nacionales en la construcción de modelos macroeconométricos puedan compartirse e intercambiarse, y apoyar los esfuerzos nacionales con asistencia regional e internacional, con el objeto de adoptar los mismos procesos desde una perspectiva de corte "transversal" de series de tiempo a nivel internacional. En algunos intentos de investigación, debería analizarse la interdependencia entre países y modelos de política de desarrollo capaces de establecer los beneficios de cooperación internacional, tal vez mediante la utilización del método demostrado en los países de América Central.

\section{BIBLIOGRAFIA}

I. Adelman y C.T. Morris, 1968, "An Econometric Model of Development". American Economic Review, 58, 5, Pt. 1, 1184-1218.

D. Agarwala, 1970, An Econometric Model of India, 1948-61, Londres: Frank Cass.

M. Brown, 1966, On the Theory and Measurement of Technical Change, Cambridge, University Press, Cap. 10.

H. B. Chenery y A.M. Strout, 1966, "Foreign Assistance and Economic Development", American Economic Review, 56, 679-733.

y L.J. Taylor, 1968, "Development Patterns: Among Countries and over Time", Review of Economics and Statistics, 50, 391-416.

B.I. Cohen y D.G. Sisler, 1971, "Exports of Developing Countries in the 1960s", Review of Economics and Statistics, 53, n. 4, 354-161.

A.B. del Río y L.R. Klein, 1973, "Macroeconometric Model Building in Latin America: The Mexican Case", en N. Ruggles, (Comp.).

B.A. De Vries, 1967, The Export Experience of Developing Countries, Washington, D.C.I.B.R.D. Agencia de Planificación Económica, Gobierno de Japón, 1965; y Econometric Models for Medium-term Economic Plan, 1964-68, Tokyo.

M. Evans, 1970, "An Econometric Model of the Israeli Economy, 1952-1953", Econometrica 38, Núm. 5, 624-660.

R.C. Fair y D.H. Jaffee, 1972, "Methods of Estimation for Markets in Disequilibrium", Econometrica 40, 497-514.

y H.H. Kelejian, 1974, "Methods of Estimation for Markets in Disequilibrium: A Further Study", Econométrica 42, Núm. 1, 177-190.

T. Fukuchi, 1973, "An Econometric Model for the Indonesian Economy", mimeo.

IE.E. Hagen y O. Hawrylyshyn, 1969, "Analysis of World Income and Growth, 19551965", Economic Development and Cultural Change, 18 Núm. 1, Parte II, 1-96.

E.P. Holland y R.W. Gillespie, 1963, Experiments in a Simulated Underdeveloped Economy: Development Plans and Balance of Payments Policies, Cambridge, M.I.T. Press.

M. Intriligator, 1971, Mathematical Optimization and Economic Theory, Englewood Cliffs, N.J.: Prentice Hall.

N. Islam, 1965, A Short-term Model for Pakistan Economy, Labori, Karachi Dacca: Oxford University Press, Pakistan Branch.

A.C. Kelley, J.G. Williamson y R.J. Cheetham, 1972. Dualistic Economic Development, Chicago and London: University of Chicago Press.

L.R. Klein, 1965, "What Kind of Macroeconometric Model for Developing Economies?", Econometric Annual of the Indian Economic Journal, 13, Núm. 3, 
313-324, reproducido en A. Zellner (Comp.), Readings in Economic Statistics and Econometrics. Boston, Little, Brown and Co. 1968, 559-570.

A.O. Krueger, 1968, "Factor Endowments and Per Capita Income Differences among Countries", Economic Journal.

S. Kuznets, 1966, Modern Economic Growth, New Haven. Yale University Press.

G.S. Maddola y F.D. Nelson, 1974, "Maximum Likelihood Methods for Models of Markets in Disequilibrium", Econométrica 42, Núm. 6, 1013-1030.

K. Marwah, 1963, "An Econometric Model of Price Behaviour in India", disertación doctoral inédita, University of Pennsylvania.

, 1970, "An Econometric Model of Colombia: A Prototype Devaluation View", Econométrica 37, Núm. 2, 228-251.

M. Marzouk, 1969, "The Predictability of Predetermined Variables in Macroeconometric Models for Developing Economies", disertación doctoral, inédita, Universiy of Pennsylvania.

N.V.A. Narashimham, 1956, A Short-term Planning Model for India, Amsterdam, North-Holland.

S. Naya, 1968, "Variations in Export Growth among Developing Asian Countries", Economic Record, 480-495.

J.B. Nugent, 1967, "Country Study-Argentina", Summer Research Report, Office of Program Coordination, Agency for International Development.

1974, Economic Integration in Central America: Empirical Investi. gations, Baltimore, Johns Hopkins University Press.

, y M.E. DePrano, 1966, "The Effects of Long-run and Short-run Planning Goals in Economic Policy Instruments", Trabajo presentado a la Sociedad Econometrica, Washington, D.C.

V.D. Ooms, 1966, "Regionalization and Export Performance: A Study of Primary Commodities", New Haven, Yale University, disertación doctoral, inédita.

M. Shubik, 1966, "Simulation of Socio-economic Systems: An Aggregate Socioeconomic Simulation of a Latin American Country", Yale University, Cowles Foundation, Trabajo para discusión, Núm. 203, Parte II, Mimeo.

R.M. Solow, 1957, "Technical Change and the Aggregate Production Function", Review of Economics and Statistics 39, 312-320.

P.M. Sommers y D.B. Suits, 1971, "A Cross-Section Model of Economic Growth", Review of Economics and Statistics 53, Núm. 2, 121-128.

D.B. Suits, 1964, An Econometric Model of the Greek Economy, Atenas, Center of Economic Research.

P.A. Yotopoulos y J.B. Nugent, de próxima publicación, Economics of Development: Empirical Investigations, Nueva York, Harper and Row.

P. Zarembka, 1967, "A Long-run Economic Growth Model for Underdeveloped Countries", Disertación doctoral, inédita, University of Wisconsin.

, 1972, "A Long-run Economic Growth Model for Developing Countries", en L.E. DeMarco (Comp.), International Economics and Development: Essays in Honor of Raúl Prebisch, Nueva York Academic Press. 